\title{
Promoting Undergraduate Student Experiential Learning, using Advanced Microscopy and Spectroscopy Instrumentation on Common Materials
}

\author{
Nanthawan Avishai ${ }^{1}$, Kevin Abbasi ${ }^{1}$ and Amir Avishai ${ }^{1}$ \\ 1. Swagelok Center for Surface Analysis of Materials, Case Western Reserve University, Cleveland, $\mathrm{OH}$, \\ USA.
}

The Swagelok Center for Surface Analysis of Materials (SCSAM) at Case Western Reserve University has initiated a program to expand undergraduate student exposure to materials characterization methods outside their curriculum. A full day short course covering the theory behind the analytical equipment and demos are offered a few times each year. However, SCSAM has attempted to find a better to way to motivate undergraduate students to be more aware of material sciences and to engage in critical thinking. Using everyday objects is an effective strategy for promoting student engagement, fostering self-directed research, and encouraging the transition from passive learning to proactive inquiry.

In-class use of analytical instruments has commonly served only a small numbers of students due to the cost, limitations of access, and need for advanced training. For faculty and staff, changes to classroom practices are time consuming and carry a risk of failure, which can impede the spread of promising educational strategies. In addition, students might lack motivation since the samples analyzed are not of their choice or interest.

To overcome these barriers SCSAM has provided funding for a pilot program developed for undergraduate education. Interactive demonstrations using commonly used items, for example, cosmetic powders (Fig. 1), and shampoo and conditioner (Fig. 2), have been used to analyze their chemical ingredients and to educate students of the role of these beauty aids.

Each project starts with a general discussion of the product that includes their function and any previous knowledge the students might have about it. SCSAM staff prepare beforehand a brief summary about the product that is shared with the students. Each student is asked to bring a sample of their choice that is then analyzed for $20-30 \mathrm{~min} /$ student. The experiments are followed by a discussion where the students share their experience and what they have learned.

Initial qualitative assessments of the initiative indicate an improvement in undergraduate motivation to learn about characterization and link to their research projects. The combination of this type of exposure together with laboratory demonstrations, where students get a more limited hands on exposure, can enhance the understanding of the relationship between the structure and performance of the materials, and lead to real problem-solving skills. Just as important are the long-term benefits, which include increasing the student's enthusiasm towards science and greater willingness to undertake graduate level research. 

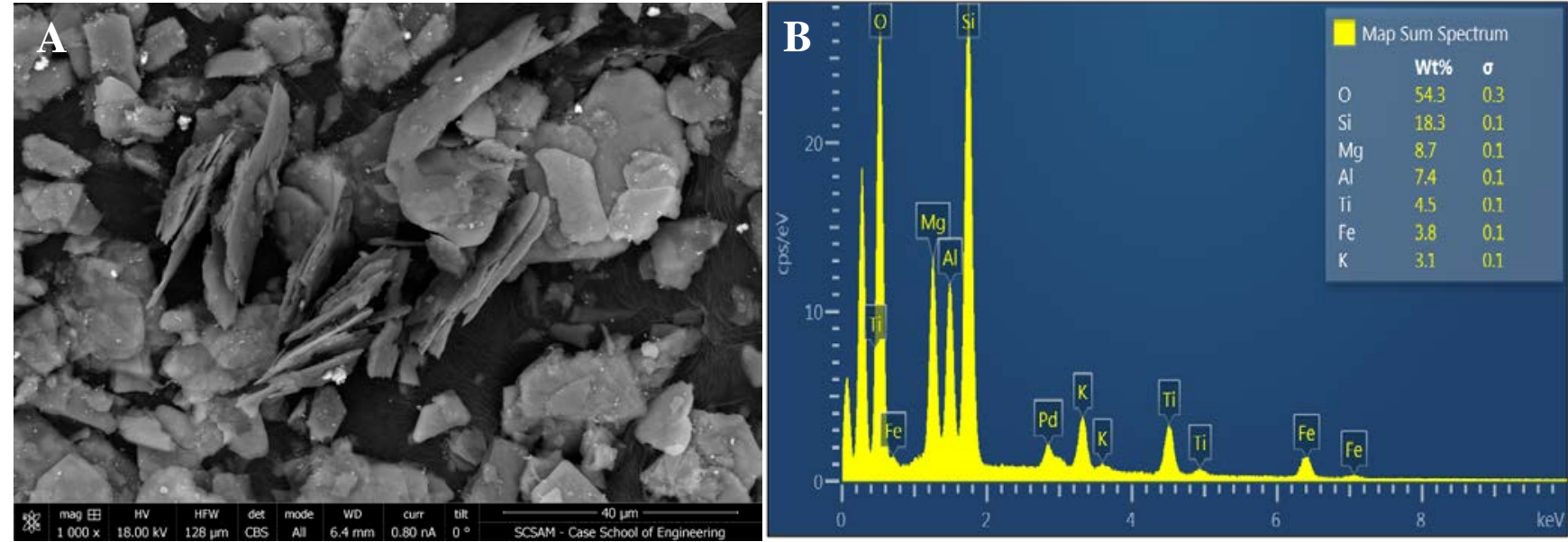

Figure 1. BSE images of a cosmetic product (A), and EDS analysis (B). The SEM images were taken with a FEI Helios Nanolab 650 using CBS at $18 \mathrm{kV}$ and $0.8 \mathrm{nA}$. The XEDS analysis was performed using an Oxford X-Max 80mm detector.
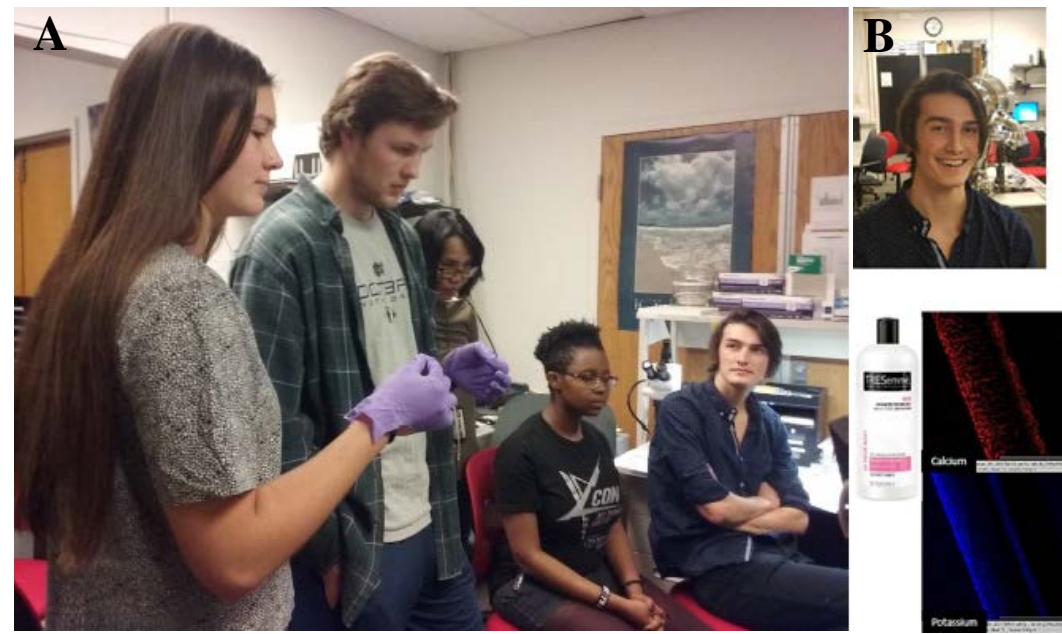

Comparing hair conditioner and shampoo products on hair with Time of flight secondary mass spectrometry - TofSIMS Wyatt Sainclair undergraduate student

Figure 2. Undergraduate students participated in analyzing their hair using TOF-SIMS: Comparison of hair strands before (A) and after applying shampoo and conditioner (B). 